\title{
Ethnic Differences in the Management of Coronary Heart Disease Patients: Lessons to Be Learned in Indo-Asians
}

\author{
Maqsood Elahi Govind Chetty Bashir Matata \\ Department of Cardiothoracic Surgery, Glenfield General Hospital, Leicester, UK
}

\section{Key Words}

Ethnicity $\cdot$ Cardiovascular disease $\cdot$ Ischaemic heart disease $\cdot$ Stroke

\begin{abstract}
Objectives: To examine the impact of ethnicity on the outcome of coronary artery bypass grafting (CABG) for myocardial revascularization. Subjects and Methods: We evaluated retrospectively 7,226 Caucasian (CC) and 650 Indo-Asian (IA) patients who underwent isolated first-time CABG surgery over 5 years. The relationship between ethnicity (IA vs. CC) and 30-day mortality, 6month mortality and complications were analysed. Results: After adjustment for baseline characteristics, IA and CC patients had similar 30-day [IA/CC odds ratio (OR) $1.07 ; 95 \%$ confidence interval $(\mathrm{Cl}) 0.84-1.35 ; \mathrm{p}=0.59]$ and 6-month mortality risk (IA/CC OR 1.10; 95\% Cl 0.91-1.34; $p=0.31)$. However, IA patients were more likely to experience complications following surgery (OR $1.28 ; 95 \% \mathrm{Cl}$ $1.14-1.45 ; p<0.01)$. Conclusion: Ethnicity does not appear to be a strong risk factor for adverse outcomes following CABG surgery in the IA patients. However, future studies are needed to elucidate the specific reasons for higher complication rates in IA patients and to devise strategies to reduce complications following the operation for these patients.
\end{abstract}

\section{KARGER}

Fax +4161306 1234 E-Mail karger@karger.ch www.karger.com

\section{Introduction}

People of non-European origin form around 7\% of the total UK population. Most of these are of Indo-Asian or Black African descent. For these migrants, as for virtually all population groups living in the western world, cardiovascular disease is the main cause of death. But there are striking ethnic differences in the risk of cardiovascular disease. Disease presentation may differ, challenging diagnostic skills, and therapeutic requirements and responses may not be uniform either. The study of ethnic differences in cardiovascular disease has provided valuable aetiological clues, not just for ethnic minority groups, but also for the majority population.

Migrants of Indo-Asian (IA) descent worldwide have elevated risks of morbidity and mortality because of ischaemic heart disease (IHD) [1]. In the UK, mortality from IHD in both IA men and women is 1.5 times that of the general population [2], and IAs have not benefited to the same extent from the general decline in deaths caused by IHD over the last few decades.

These ethnic differences have been extensively studied, and classical risk factors, such as smoking, blood pressure, obesity and cholesterol, vary substantially between IA and Caucasian (CC) populations. Studies have also suggested that a non-CC ethnic group in general is associated with lower rates of cardiovascular procedure use, including coronary artery bypass grafting $(\mathrm{CABG})$ with fewer reported complications postoperatively $[3,4]$. 
Though the influence of race on cardiovascular procedure use and outcomes has been an area of extensive investigation, the impact of ethnicity on outcomes following coronary revascularization is less clear. Hence, we aim to study 30-day and 6-month mortality and 30-day complication rates, following $\mathrm{CABG}$ for IA patients in comparison to $\mathrm{CC}$ patients.

\section{Subjects and Methods}

\section{Study Population}

A retrospective review of 7,876 charts was carried out in the fall of October 1999 to October 2004 for patients who underwent isolated first-time CABG surgery on cardiopulmonary bypass. All the patients operated as elective or urgent $\mathrm{CABG}$ were included. Patients who had undergone heart surgery previously and those with associated valvular disease were excluded. If a degree of valvular dysfunction was suspected before surgery, a peri-operative transoesophageal echocardiogram was performed. Therefore, only patients with coronary artery disease and without significant valvular disease were included in the study. Age, sex, ethnicity (IA vs. CC) and most recent weight, height, glucose, cholesterol and lipid profiles were obtained from the charts of 650 patients of IA descent and 7,226 CC patients.

\section{Risk Stratification}

All patients were prospectively risk stratified utilizing EuroSCORE pre-operatively. Using the calculated EuroSCORE values, the patients were thus classified into three groups: low-risk group, $\leq 2$; moderate-risk group, $>2$ but $<6$; high-risk group, $\geq 6$.

The mean EuroSCORE value was indicative of the overall expected mortality within the retrospective risk groups or the patient population as a whole.

\section{Data Collection}

Baseline demographic and clinical data were available for all the patients. The pre-operative medications, including $\beta$-blockers, angiotensin-converting enzyme inhibitors and calcium antagonists, were continued up to the day of surgery, except acetylsalicylic acid that was discontinued at least 1 week before surgery. The outcomes of the IA CABG patients, namely total number of grafts performed, type of grafts used, duration of postoperative intensive-care unit stay and hospital stay and postoperative complications, were recorded and comparisons were made with respect to outcomes of the CC CABG patients. Patients were followed for a period of 6 months after the procedure. Mortality was taken into account if it occurred within 30 days after the procedure.

\section{Variables}

The three outcome variables for this study were: 30-day allcause mortality, 6-month all-cause mortality and 30-day complication rates. Mortality assessments were done using a computerized patient record system shown to be comparable with the National Death Index for mortality assessment. Complications were assessed by chart review that included reoperative bleeding, pulmonary oedema, pulmonary effusion, pneumothorax, renal failure, renal impairment, dialysis, urinary retention, neuropraxia, tempo- rary/permanent stroke, delirium, limb ischaemia, arrhythmias (atrial flutter, atrial fibrillation, bradycardia), postoperative myocardial infarction, sternal wound infection, leg wound infection, pyrexia and pneumonia. The primary predictor variable for this study was ethnicity (IA and CC). A wide array of pre-operative variables, including demographic, cardiac and non-cardiac factors, as well as intra-operative variables such as number and types of grafts used, were available for risk adjustment (table 1). These variables included established risk variables for 30-day and 6-month mortality and 30-day complications $[5,6]$.

\section{0-Day and 6-Month Mortality}

The unadjusted association between IA versus $\mathrm{CC}$ ethnicity and both 30-day and 6-month mortality were evaluated using $\chi^{2}$ tests. Multiple linear regression models were then developed to assess the impact of IA ethnicity on 30-day and 6-month mortality after adjusting for all of the variables listed in table 1. Interactions between ethnicity and several risk factors shown to have different prevalences between ethnicities, including age, diabetes and renal insufficiency, were added to the logistic regression models to determine their significance. We chose to report the logistic regression results for comparability to the 30-day mortality models. Furthermore, given the relatively low risk of death and the lack of censoring in this study population (complete follow-up on all patients), Cox regression is not more efficient than logistic regression [7], and logistic regression allows reporting of odds ratios (ORs) that are easily interpretable.

\section{Statistical Analysis}

The data collection and subsequent statistical analysis were performed with MS Excel for Windows 2000 and SPSS for Windows. The objective of this study was to determine whether IA patients are at a different risk for adverse outcomes compared with $\mathrm{CC}$ patients following CABG surgery. Therefore, $\mathrm{CC}$ ethnicity was the referent group for all analyses, and comparisons were made. Baseline characteristics of the study population were compared between IA and CC patients using $t$ tests for continuous variables and $\chi^{2}$ tests for categorical variables. Statistical tests of significance were performed, and a $\mathrm{p}$ value $<0.05$ was assumed to be statistically significant.

\section{Results}

\section{Baseline Characteristics}

Baseline characteristics of the study population comparing IA and CC patients are shown in table 1. Compared with CC patients, IA patients were more likely to have prior percutaneous coronary intervention, S-T segment depression on pre-operative electrocardiogram, elevated serum creatinine, peripheral vascular disease, diabetes, to be current smokers and to receive pre-operative diuretics. Conversely, $\mathrm{CC}$ patients were more likely than IA patients to have prior heart surgery, chronic obstructive pulmonary disease and to be classified as having urgent or emergent surgery. Overall, there was no significant difference 
Table 1. Characteristics of the study population

\begin{tabular}{|c|c|c|c|}
\hline Variables & $\begin{array}{l}\text { Caucasian } \\
(\mathrm{n}=7,226)\end{array}$ & $\begin{array}{l}\text { Indo-Asian } \\
(\mathrm{n}=650)\end{array}$ & $\mathrm{p}$ value \\
\hline \multicolumn{4}{|l|}{ Demographics } \\
\hline Age (mean $\pm S D)$, years & $63.4 \pm 9.4$ & $62.2 \pm 10.0$ & 0.31 \\
\hline Gender, \% male & 92.3 & 93.1 & 0.91 \\
\hline $\mathrm{BSA}($ mean $\pm \mathrm{SD}), \mathrm{m}^{2}$ & $2.01 \pm 0.2$ & $2.00 \pm 0.2$ & 0.10 \\
\hline \multicolumn{4}{|l|}{ Cardiac, \% } \\
\hline Prior MI & 58.4 & 56.4 & 0.05 \\
\hline Prior PCI & 1.7 & 2.8 & $<0.01$ \\
\hline Prior CABG & 7.6 & 4.2 & $<0.01$ \\
\hline Pre-op. IABP & 5.4 & 4.9 & 0.36 \\
\hline CCS III or IV & 80.5 & 77.5 & $<0.01$ \\
\hline NYHA III or IV & 3.5 & 3.9 & 0.21 \\
\hline LVEF <30\% & 11.5 & 12.7 & 0.34 \\
\hline 3-vessel CAD & 71.8 & 73.3 & 0.11 \\
\hline \multicolumn{4}{|l|}{ Pre-op. S-T segment } \\
\hline depression & 19.1 & 23.7 & $<0.01$ \\
\hline Pre-op. diuretics & 23.9 & 27.4 & $<0.01$ \\
\hline \multicolumn{4}{|l|}{ Pre-op. intravenous } \\
\hline nitroglycerin & 14.9 & 12.9 & $<0.01$ \\
\hline \multicolumn{4}{|l|}{ Surgery priority, \% } \\
\hline Elective & 82.4 & 86.5 & \\
\hline Urgent & 11.5 & 9.6 & \\
\hline Emergent & 6.1 & 3.9 & \\
\hline ITA used & 82.4 & 82.8 & 0.67 \\
\hline Grafts (mean \pm SD) & $3.18 \pm 0.97$ & $3.10 \pm 1.01$ & $<0.01$ \\
\hline
\end{tabular}

\section{Non-cardiac}

Serum creatinine

\begin{tabular}{lllr}
$\quad$ (mean \pm SD), mg/dl & $1.22 \pm 0.7$ & $1.43 \pm 1.2$ & $<0.01$ \\
PVD, \% & 23.5 & 22.4 & 0.23 \\
CVD, \% & 21.5 & 19.8 & 0.05 \\
Diabetes mellitus, \% & 31.4 & 38.1 & $<0.01$ \\
Current smoker, \% & 31.6 & 34.9 & $<0.01$ \\
COPD, \% & 25.9 & 19.6 & $<0.01$ \\
Hypertension, \% & 82.6 & 90.0 & $<0.01$ \\
\hline Postop. stay (mean \pm SD), days & & \\
ICU & $1.74 \pm 1.32$ & $1.37 \pm 0.95$ & 0.31 \\
Hospital & $7.88 \pm 3.84$ & $7.03 \pm 2.34$ & 0.09 \\
\hline Mortality, \% & & \\
30-day & 3.6 & 3.9 & 0.48 \\
6-month & 5.8 & 6.3 & 0.27 \\
\hline
\end{tabular}

$\mathrm{BSA}=$ Body surface area; $\mathrm{CAD}=$ coronary artery disease; $\mathrm{CCS}=$ Canadian Cardiovascular Society COPD = chronic obstructive pulmonary disease; $\mathrm{CVD}=$ cerebrovascular disease; $\mathrm{IABP}=$ intra-aortic balloon pump; ITA = internal thoracic artery; LVEF = left-ventricular ejection fraction; $\mathrm{MI}=$ myocardial infarction; NYHA = New York Heart Association; PCI = percutaneous coronary intervention; PVD = peripheral vascular disease. in the estimated pre-operative mortality risk between IA and $\mathrm{CC}$ patients. There was little difference in the way the outcomes of the two ethnic groups differed between the three risk categories. However, IA patients were more likely to have 1 or more complications within 30 days following surgery in the moderate-risk category whereas in the high-risk category IA patients seemed to bleed more and had more pulmonary, vascular and cardiac related complications as compared to CC patients (table 2).

\section{Risk-Adjusted Outcomes}

In the regression models shown in table 3, there were no significant differences in overall risk-adjusted 30-day [OR 1.07; 95\% confidence interval (CI) 0.84-1.35; p = 0.59 ) or 6-month mortality (OR 1.10; 95\% CI 0.91-1.34; $\mathrm{p}=0.31$ ) between IA and CC patients. There was no significant interaction between IA ethnicity and age, diabetes or serum creatinine in the regression models. However, IA patients were more likely to have 1 or more complications within 30 days after the operation (OR 1.28; 95\% CI 1.14-1.45; p < 0.01), after adjustment for baseline characteristics.

\section{Discussion}

The potential importance of the aetiology of coronary heart disease in IA has prompted researchers to explore clues as to the exact mechanisms of such an association. Attention has focused on inflammation, infection, haemostatic factors and endothelial dysfunction. C-reactive protein concentrations are elevated in IA, caused at least in part by a greater degree of central adiposity which promotes C-reactive protein expression [8]. C-reactive protein is highly correlated with fasting and postload insulin and independently predicts the risk of IHD. PAI-1 and homocysteine concentrations are also raised in IAs, and endothelial function is impaired; these may also contribute to the elevation in IHD risk, either related to or separately from their associations with insulin resistance $[9,10]$.

Genetic predisposition to insulin resistance could be postulated [11, 12], although no factor has as yet been isolated. Poor in utero development, resulting in low birth weight, in particular coupled with catch-up growth in early childhood, has been associated with a greater prevalence of insulin resistance in IAs [13]. Other factors, separate from insulin resistance, which may further account for the ethnic susceptibility to IHD include lipoprotein $\mathrm{Lp}(\mathrm{a})$, concentrations of which are thought to be largely genetically determined. Circulating $L p(a)$ is substantially 
Table 2. Complications and mortality between CC and IA patients under different risk categories

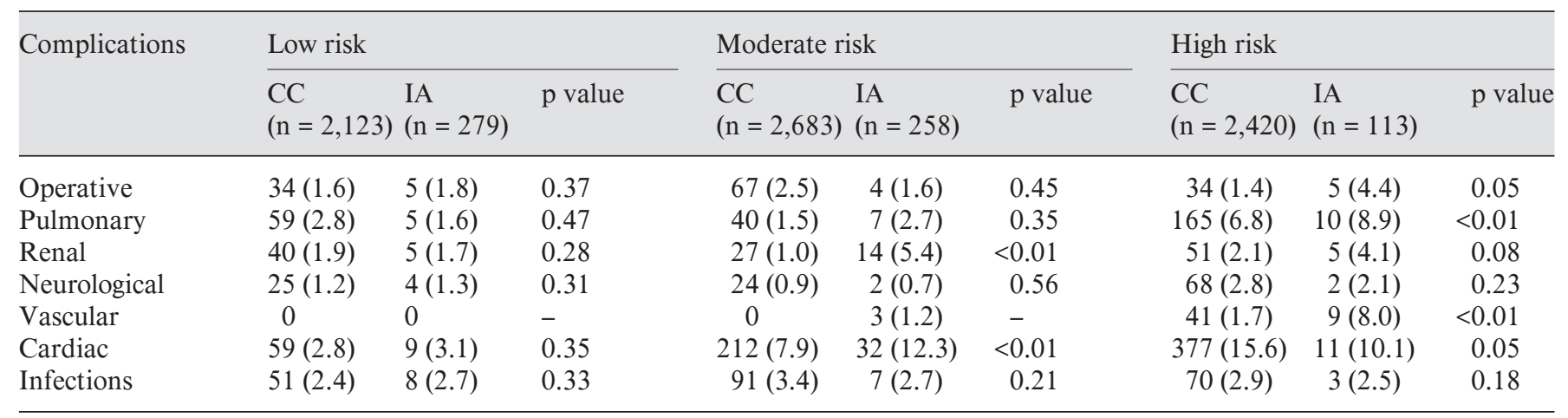

Results are expressed as numbers of patients, with percentages in parentheses. Operative = reoperative bleeding; pulmonary = pulmonary oedema, effusion, pneumothorax; renal = renal failure, renal impairment, dialysis, urinary retention; neurological = neuropraxia, temporary/permanent stroke, delirium; vascular = limb ischaemia; cardiac = arrhythmias (atrial flutter, atrial fibrillation, bradycardia), postoperative myocardial infarction; infections = sternal wound, leg wound, pyrexia, pneumonia.

Table 3. Multivariate risk models for 30-day mortality, 6-month mortality and 30-day complications

\begin{tabular}{lll}
\hline Variables & OR & p value \\
\hline 30-day mortality & & - \\
CC & 1.0 & 0.59 \\
IA & $1.07(0.84-1.35)$ & - \\
\hline 6-month mortality & 1.0 & 0.31 \\
CC & $1.10(0.91-1.34)$ & \\
IA & & - \\
\hline 30-day complications & 1.0 & $<0.01$ \\
CC & $1.28(1.14-1.45)$ & \\
IA &
\end{tabular}

Figures in parentheses indicate confidence intervals.

raised in IA compared to CC individuals [11], and, separately, raised $L p(a)$ has been shown to increase the risk of IHD events, especially in the presence of raised low-density lipoprotein cholesterol.

However, this does not mean that conventional risk factors are unimportant in IAs. The risk of IHD in current smokers, for example, is 4 times that of a never smoker in a given IA population, a similar elevation in risk to that observed in CCs. Thus, within IAs, conventional risk factors are of immense importance in predicting absolute risks of IHD, but factors accounting for differences between ethnic groups (that is, relative risks) are quite different. Because of the elevated absolute risk, and because the interrelationships between, for example, total cholesterol, high-density lipoprotein cholesterol and triglyceride may differ in IAs, conventional risk calculations may be misleading so that the threshold at which lipid-lowering therapy should be instituted may also be lower than current guidelines would suggest.

There is a widespread belief that coronary arteries are smaller in IAs. If these as an ethnic group had generally smaller coronary arteries, this may give the impression of severer disease and have clinical implications, especially for revascularization procedures, such as $\mathrm{CABG}$ and atherectomy. This indicates that the cardiologist or cardiothoracic surgeon contemplating revascularization should be prepared to encounter generally smaller coronary artery segments in IA subjects. CABG surgery would also be more difficult in view of the difficulties in anastomoses between saphenous vein grafts or internal mammary conduits to small-calibre native coronary arteries as, for any intervention, it is the real or absolute size of the coronary arteries that matters $[14,15]$.

\section{Conclusion}

The results of this study suggest that ethnicity does not appear to be a strong risk factor for adverse outcomes following CABG surgery in the IA patients. However, future studies are needed to elucidate the specific reasons for higher complication rates in IA patients and to devise strategies to reduce complications following the operation for these patients. 


\section{References}

1 McKeigue PM, Miller GJ, Marmot MG: Coronary heart disease in South Asians overseas - A review. J Clin Epidemiol 1989;42:597-609.

$>2$ Wild S, McKeigue P: Cross sectional analysis of mortality by country of birth in England and Wales, 1970-1992. BMJ 1997;314:705-710.

-3 McKeigue PM, Shah B, Marmot MG: Relation of central obesity and insulin resistance with high diabetes prevalence and cardiovascular risk in South Asians. Lancet 1991;337:382386.

$\checkmark 4$ Reaven GM: Role of insulin resistance in human disease. Diabetes 1988;37:1595-1607.

5 Jones RH, Hannan EL, Hammermeister KE, Delong ER, O'Connor GT, Luepker RV, Parsonnet V, Pryor DB: Identification of preoperative variables needed for risk adjustment of short-term mortality after coronary artery bypass graft surgery. The Working Group Panel on the Cooperative CABG Database Project. J Am Coll Cardiol 1996;28:1478-1487.
66 Rumsfeld JS, MacWhinney S, McCarthy M Jr, Shroyer AL, VillaNueva CB, O'Brien M, Moritz TE, Henderson WG, Grover FL, Sethi GK, Hammermeister KE: Health-related quality of life as a predictor of mortality following coronary artery bypass graft surgery. Participants of the Department of Veterans Affairs Cooperative Study Group on Processes, Structures, and Outcomes of Care in Cardiac Surgery. JAMA 1999;281:1298-1303.

7 Cuzick J: The efficiency of the proportions test and the log rank test for censored survival data. Biometrics 1982;38:1033-1039.

$>8$ Chambers JC, Eda S, Bassett P, Karim Y, Thompson SG, Gallimore JR, Pepys MB, Kooner JS: C-reactive protein, insulin resistance, central obesity, and coronary heart disease risk in Indian Asians from the United Kingdom compared with European whites. Circulation 2001;104:145-150.

$\checkmark 9$ Chambers JC, Obeid OA, Refsum H, Ueland P, Hackett D, Hooper J, Turner RM, Thompson SG, Kooner JS: Plasma homocysteine concentrations and risk of coronary heart disease in UK Indian Asian and European men. Lancet 2000;355:523-527.

10 Kain K, Catto AJ, Grant PJ: Impaired fibrinolysis and increased fibrinogen levels in South Asian subjects. Atherosclerosis 2001;156:457461.
11 Bhatnagar D, Anand IS, Durrington PN, Patel DJ, Wander GS, Mackness MI, Creed F, Tomenson B, Chandrashekhar Y, Winterbotham $\mathrm{M}$, et al: Coronary risk factors in people from the Indian subcontinent living in West London and their siblings in India. Lancet 1995;345: 405-409.

12 Neel JV: Diabetes mellitus: a 'thrifty' genotype rendered detrimental by 'progress'. Am J Hum Genet 1962;14:353-362.

13 Bavdekar A, Yajnik CS, Fall CH, Bapat S, Pandit AN, Deshpande V, Bhave S, Kellingray SD, Joglekar C: Insulin resistance syndrome in 8year-old Indian children: small at birth, big at 8 years, or both? Diabetes 1999;48:24222429.

14 Lip GY, Rathore VS, Katira R, Watson RDS, Singh SP: Do Indo-Asians have smaller coronary arteries? Postgrad Med J 1999;75:463466.

15 Kaimkhani Z, Ali M, Faruqui AM: Coronary artery diameter in a cohort of adult Pakistani population. J Pak Med Assoc 2004;54:258261. 